\title{
Medicago lesinsii E. Small (Leguminosae), nueva cita para la flora ibérica
}

\author{
Carlos Romero-Zarco \\ Departamento de Biología Vegetal y Ecología, Universidad de Sevilla
}

\section{Correspondencia \\ C. Romero-Zarco}

e-mail: zarco@us.es

Recibido: 12 marzo 2019

Aceptado: 10 septiembre 2019

Publicado on-line: 2 octubre 2019

Editado por: B. Cabezudo
Medicago lesinsii E. Small (Leguminosae), a new species for the Iberian flora

Palabras clave: Flora, Andalucía, Leguminosae, Medicago

Key words: Flora, Andalusia, Leguminosae, Medicago
La complejidad taxonómica y nomenclatural del género Medicago L. (Leguminosae) es bien conocida, especialmente en lo que respecta a la sección Spirocarpos Ser. y, dentro de ella, a las especies de la subsección Pachyspireae (Urb.) Heyn, caracterizadas por sus legumbres de espiras (vueltas) muy apretadas y duras en la madurez. Una de sus especies, Medicago murex Willd., poco frecuente en nuestro territorio, comprende al parecer dos taxones muy similares morfológicamente pero con números cromosómicos diferentes: $M$. murex sensu stricto $(2 n=14)$ y M. lesinsii E. Small $(2 n=$ 16). De acuerdo con Small \& Brookes (1985) las plantas de estas dos entidades no son interfértiles y pueden reconocerse morfológicamente, lo que apoya un tratamiento taxonómico específico, siendo la primera posiblemente un derivado aneuploide de la segunda (Lesins \& al., 1970). En las bases de datos se incluye el nombre de Small en la sinonimia de M. murex (cf. ILDIS, 2018). Sin embargo, no se menciona en ninguna de las floras básicas del proyecto Flora iberica (Castroviejo, 1986-2012; Aedo, 2013-2019), ni tampoco en el tratamiento que Sales \& Hedge (1999) hicieron para el género en dicha obra. De acuerdo con Small \& Brookes (op. cit.), otros nombres específicos o infraespecíficos que pudieron ser investigados fueron identificados con el tipo de M. murex, taxón más vigoroso y extendido.

Bajo estas premisas se ha investigado el material andaluz conservado en los herbarios MCG y SEV, como paso previo a la revisión de otros materiales peninsulares, constatando la presencia de las dos especies en Andalucía, resultando ser M. lesinsii nueva especie para el catálogo andaluz y por tanto también para el peninsular.
Medicago murex Willd., Sp. Pl. 3: 1410 (1802)

M. globulifera Desf. ex Poir. in Lam., Encycl. Suppl. 3: 526 (1813)

M. ovata Carmign. in Giorn. Pis. 5(12): 136 (1810), quod descriptio

M. sicula Tod., Ind. Sem. Panorm. 1868: 27 (1868), quod descriptio

M. sphaerocarpa Bertol., Rar. Lig. PI. Decas Prima 3: 60 (1810)

Indicación locotípica: no la hay [tipo en B (cf. Small \& Brookes, 1985)]

Iconografía: J. Borja, Mielgas Carretones Españ.: 35 fig. A (1962); E. Small \& B.S. Brookes in Canadian Journal of Botany 63: 731 fig. 2 (1985).

Diagnosis. Hierba anual, glabra salvo algunos pelos dispersos en el envés de los folíolos. Folíolos de las hojas medias y superiores obovadocuneados, obtuso-mucronados o ligeramente emarginado-mucronados, con 30-42 dientes (serrulaciones) en la parte distal (Fig. 1 a, b). Racimos con 2-4 flores. Legumbre turbinada, de espiras apretadas, con espinas (en el material ibérico); espiras con una banda submarginal lisa en la que confluyen los nervios laterales; margen de la legumbre madura claramente trinervado, con los nervios formando sendas crestas semejantes en altura.

Distribución conocida. Región mediterránea.

Material estudiado

Cádiz. Algeciras, km 99 N 340, 250 m, 23-IV1980, J. Arroyo \& al. $n^{\circ}$ X 731/80 (SEV 67112); íbidem, Sierra del Algarrobo, garganta, $200 \mathrm{~m}$, 29-IV-1981, J. Arroyo \& J.M. Gil n ${ }^{\circ}$ X 2605/81 (SEV 68055); Los Barrios, Sierra de la Palma, Las Zorrillas, Arroyo del Tiradero, 200 m, 10-V-1980, J. Arroyo et al. (SEV 67265); Medina Sidonia, Puerto 
de los Reyes, márgenes del pantano del Celemín, TF 5021, 20 m, 2-V-1980, E. Díez, C. Romero \& S. Silvestre (SEV 125096).

Observaciones. La especie se reconoce sobre todo porque sus legumbres maduras muestran los nervios laterales (poco manifiestos) confluyendo en una banda submarginal lisa que ocupa c. 1/3 de su diámetro, sin nervios; otras especies de la misma sección presentan en su lugar un nervio paralelo al margen (nervio submarginal). Este carácter es compartido solamente con $M$. turbinata (L.) All. que, a diferencia de M. murex, es hierba pelosa. Por lo que sabemos, todo el material estudiado de la Península y Baleares presenta legumbres espinosas (Borja, 1962; Sales \& Hedge, 1999). En Flora iberica se indican para Andalucía las provincias de Cádiz y Sevilla en base a testimonios de herbario, y de Jaén por referencia bibliográfica (Sales \& Hedge, op. cit.). Las plantas de la provincia de Sevilla que han podido ser estudiadas (revisadas por dichos autores en 1993) pertenecen a $M$. lesinsii y no a $M$. murex (véase más abajo). La referencia bibliográfica para Jaén debe corresponder a la cita de Willkomm (1877: 385): "regni Jienn. (pr. Jaen, LGE.)"; la misma que justificó la inclusión de esta especie en Flora Vascular de Andalucía oriental (Romero-Zarco,
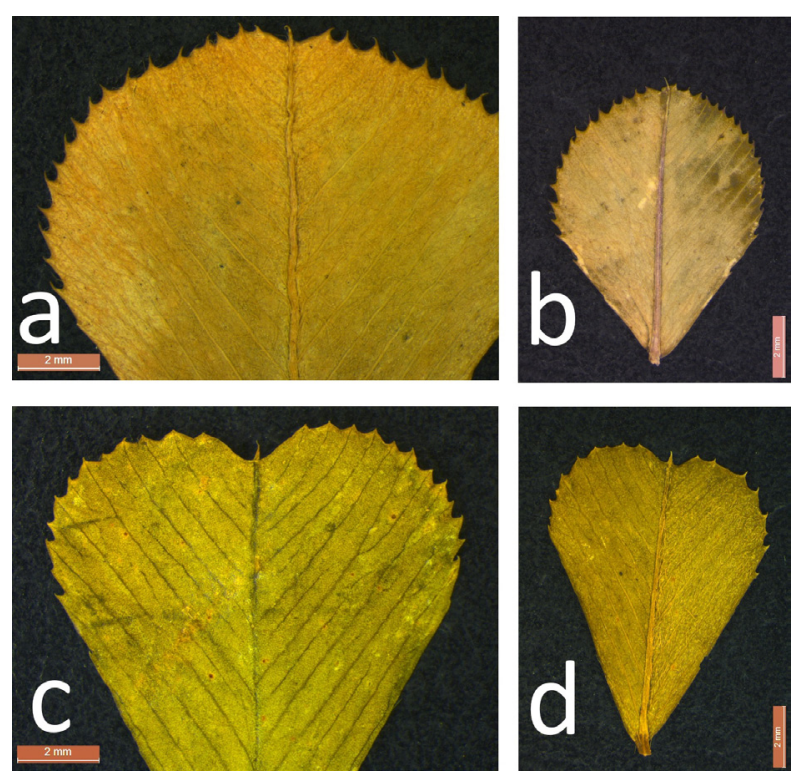

Figura 1. Ejemplos de folíolos de las hojas medias (a, c) y distales (b, d) de Medicago murex (a, b) y M. lesinsii (c, d). a) M. murex, SEV 125096; b) M. murex, SEV 67265; c) M. lesinsii, SEV 22671; d) M. lesinsii, SEV 288087.

Figure 1. Examples of leaflets from middle $(a, c)$ and distal leaves $(b, d)$ of Medicago murex $(a, b)$ and $M$. lesinsii $(c, d)$.
2009) según consta en mi archivo. En la monografía de Borja (1962) lo que se dibuja y describe es claramente esta especie y las plantas que se citan son del sur de Cádiz. Por el contrario en la revisión de Casellas (1962: 246) se desestiman las citas andaluzas para esta especie.

Medicago lesinsii E. Small in Canad. J. Bot. 63: 733 (1985)

Holotipo: "E. Small M1149 (DAO), grown in Ottawa greenhouse, 1983-4, from seeds collected by A. Abdelguerfi (174f), Dra el Alag. Djebel Ouahche (Constantine), Algeria."

Iconografía: E. Small \& B.S. Brookes in Canadian Journal of Botany 63: 732 fig. 3 (1985); C. Romero Zarco in Valdés \& al. (eds.), Flora Vascular de Andalucía Occidental 2: 124 (1987), sub M. murex.

Diagnosis. Hierba anual, glabra salvo algunos pelos dispersos en el envés de los folíolos. Folíolos de las hojas medias y superiores largamente cuneados, emarginado-mucronados, con 12-26 dientes (serrulaciones) en la parte distal (Fig. $1 \mathrm{c}$, d). Racimos con 1-2 flores. Legumbre turbinada, de espiras apretadas, frecuentemente con espinas (tipo), rara vez sin ellas; espiras con una banda submarginal lisa en la que confluyen los nervios laterales; margen de la legumbre madura plano y casi liso, con los nervios apenas salientes.

Distribución conocida. Argelia, Cerdeña, España (novedad) e Israel.

Material estudiado

Cádiz. Ubrique, garganta de Barrida, casa de la Patilla, TF 8258, 300 m, 9-VI-1983, A. Aparicio \& al. (SEV 241104). Huelva. El Cerro del Andévalo, a $25 \mathrm{~km}$ de Cabezas Rubias, en el cruce AyamonteTharsis, pastizal bajo encinar, 29S PB 6775, 6-V1990, K. Ferguson, C. Santa-Bárbara \& B. Valdés $\mathrm{n}^{\circ}$ SB 857 (SEV 288086); ídem $\mathrm{n}^{\circ} \mathrm{SB} 858$ (SEV 288087). Puebla de Guzmán: márgenes del río Malagón, PB5873, 6-V-1990, K. Ferguson, C. Santa-Bárbara \& B. Valdés (SEV 144397). Málaga. Cortes de la Frontera, P.N. Los Alcornocales, EI Ramblazo, 30S TF 7444 , pastizal húmedo en margas, 18-V-1999, D. Navas, P. Navas \& Y. Gil (MGC 56622). Sevilla. Brenes, 30-V-1972, D. Cañete \& E. Conde (SEV 22670); Castilleja de la Cuesta, IV-1956, A. Peiró (SEV 7740); Isla Mayor, 9-V-1968, V.H. Heywood \& B. Valdés no 1611/68 (SEV 22669); Puebla del Río, 7-V-1972, D. Cañete \& E. Conde (SEV 22671).

Observaciones. El material andaluz presenta algunas de las características diferenciales con respecto a $M$. murexindicadas por Small \& Brookes (1985), en especial en lo referente a la morfología de los folíolos (Fig. 1) y de las legumbres. Otros 
caracteres, como son los racimos con una sola flor o las estípulas con un número menor de lacinias son menos evidentes. Solo una de las muestras observadas presenta legumbres inermes (SEV 288087) pero en la misma población había otras plantas con legumbres espinosas (SEV 288086). El pliego de Ubrique (SEV 241104) es el testimonio de una cita de $M$. murex para el Parque Natural de Grazalema (Aparicio \& Silvestre, 1987). El pliego de Puebla de Guzmán (SEV 14439) fue publicado como novedad para la provincia de Huelva sub $M$. murex (Santa-Bárbara et al., 2003).

\section{Agradecimientos}

Trabajo parcialmente financiado por el Plan Andaluz de Investigación de la Junta de Andalucía (RNM 204). Se agradece la colaboración prestada por los conservadores de los herbarios MGC (Universidad de Málaga) y SEV (Universidad de Sevilla), doctores J. García Sánchez y F.J. Salgueiro, respectivamente, así como al resto del personal de dichas instituciones.

\section{Referencias}

Aedo, C. (coord. gen., 2013-2019). Flora iberica 9, 16, 20. Real Jardín Botánico, CSIC, Madrid.

Aparicio, A. \& Silvestre, S. (1987). Flora del Parque Natural de la Sierra de Grazalema. Junta de Andalucía,
Agencia de Medio Ambiente, Monografías del Medio Ambiente, Sevilla.

Borja, J. (1962). Las "mielgas" y "carretones" españoles. Instituto Nacional de Investigaciones Agrarias. Madrid.

Casellas, J. (1962). El género Medicago L. en España. Collectanea Botanica (Barcelona) 6: 183-291.

Castroviejo, S. (coord. gen., 1986-2012). Flora iberica 1-8, 10-15, 17-18, 21. Real Jardín Botánico, CSIC, Madrid.

ILDIS (2018). International Legume Database \& Information Service; https://ildis.org/ (consultado el 9-03-2019).

Lesins, K.A., I. Lesins \& B. Gillies (1970). Medicago murex with $2 n=16$ and $2 n=14$ chromosome complements. Chromosoma 30: 109-122.

Romero-Zarco, C. (2009). Medicago L. En: G. Blanca \& al. (eds.), Flora Vascular de Andalucía Oriental 2: 417427. Junta de Andalucía, Sevilla.

Sales, F. \& I.C. Hedge (1999). Medicago L. En: S. Talavera \& al. (eds.), Flora iberica 7: 741-775. Real Jardín Botánico, CSIC, Madrid.

Santa-Bárbara, C., B. Valdés \& F.J. Pina (2003). Novedades corológicas para la comarca del Andévalo (provincia de Huelva, SO de España). III. Lagascalia 23: 91-122.

Small, E. \& B.S. Brookes (1985. Medicago lesinsii a new Mediterranean species. Canadian Journal of Botany 63: 728-724.

Willkomm, H.M. (1877). Papilionaceae L. En: H.M. Willkomm \& J.M.Ch. Lange, Prodromus Florae Hispanicae 3(2): 247-470. Stutgartiae. 